\title{
Análise clínica e sociodemográfica de pessoas com estomias: estudo transversal
}

\author{
Clinical and sociodemographic analysis of people with ostomies: a cross-sectional study
}

Análisis clínico y sociodemográfico de personas con estomas: estudio transversal

Ravena Rieelly Araujo Moura', Eliete Albano Azevedo Guimarães², Juliano Teixeira Moraes²

ORCID IDS

Moura RRA ID https://orcid.org/0000-0002-2725-6167

Guimarães EAA (iD https://orcid.org/0000-0001-9236-8643

Moraes JT (D) https://orcid.org/0000-0002-1109-962X

\section{COMO CITAR}

Moura RRA, Guimarães EAA, Moraes JT. Análise clínica e sociodemográfica de pessoas com estomias: estudo transversal. ESTIMA, Braz. J. Enterostomal Ther., 16: e3818. https://doi. org/10.30086/estima.v16.637 PT

\section{RESUMO}

Objetivos: Analisar aspectos clínicos e sociodemográficos de pessoas com estomias no estado de Minas Gerais. Métodos: Estudo de corte transversal realizado em 54 municípios, em 2016. Os dados foram coletados de 418 pessoas com estomia cadastradas a partir de um questionário estruturado. Realizou-se a análise descritiva dos dados, o teste de qui-quadrado de Pearson e a correção de Bonferroni. Resultados: A maioria das pessoas com estomias é idosa, do sexo masculino, tem baixa escolaridade e baixa renda salarial. A neoplasia maligna colorretal foi a principal causa e 14,3\% são tratados com quimioterapia. Parte das estomias é do tipo colostomias permanentes com forma regular. Destaca-se, como principal complicação, a dermatite, e 45,5\% não estão aptos a desenvolver o autocuidado. O tipo de estomia associou-se significativamente com a permanência da estomia, o estado civil e com a renda ( $<$ <,05). Conclusão: As pessoas com estomias necessitam de assistência relacionada à orientação para o autocuidado, prevenção e tratamento de suas complicações.

DESCRITORES: Estomia; Perfil de saúde; Serviços de saúde; Avaliação em saúde; Enfermagem; Estomaterapia.

\footnotetext{
${ }^{1}$ Universidade Federal de São João del-Rei - Contagem/MG - Brasil.

universidade Federal de São João del-Rei - Curso de Enfermagem - Programa de Pós-Graduação - Divinópolis/MG - Brasil. Autor correspondente: Ravena Rieelly Araujo Moura | Rua Mopage, 307 - Novo Eldorado | CEP: 32341-200 - Contagem/MG - Brasil | E-mail: ravena.araujo@yahoo.com.br Recebido: Ago 142018 | Aceito: Dez 152018
} 


\begin{abstract}
:
Objectives: To analyze clinical and sociodemographic aspects of people with ostomies in the state of Minas Gerais. Methods: A crosssectional study was conducted in 54 municipalities in 2016. Data were collected from 418 people with ostomies, registered from a structured questionnaire. Descriptive data analysis, the Pearson chi-square test and the Bonferroni correction were performed. Results: The majority of people with ostomies are elderly, male, have low schooling and low wage income. Colorectal malignant neoplasia was the main cause and $14.3 \%$ were treated with chemotherapy. Part of the ostomies is of the regular standing permanent colostomy type. The main complication highlighted is dermatitis, and $45.5 \%$ are not able to develop self-care. The type of ostomy was significantly associated with stomatal permanence, marital status and income ( $p$ <.05).Conclusion: People with ostomies need assistance related to self-care orientation, prevention and treatment of their complications.
\end{abstract}

DESCRIPTORS: Ostomy; Health profile; Health services; Health evaluation; Nursing; Stomatherapy.

\title{
RESUMEN
}

Objetivos: Analizar aspectos clínicos y sociodemográficos de personas con estomas en el estado de Minas Gerais. Métodos: Estudio de corte transversal realizado en 2016 en 54 municipios. Los datos fueron recolectados de 418 personas con estoma registrados a partir de un cuestionario estructurado. Se realizó el análisis descriptivo de los datos, la prueba de chi-cuadrada de Pearson y la corrección de Bonferroni. Resultados: La mayoría de las personas con estomas es anciana, de sexo masculino, tiene baja escolaridad y baja renta salarial. La neoplasia maligna colorrectal fue la principal causa y $14,3 \%$ son tratados con quimioterapia. Parte de las estomas es del tipo colostomías permanentes con forma regular. Se destaca, como principal complicación, la dermatitis, y 45,5\% no están aptos para desarrollar el autocuidado. El tipo de estoma se asoció significativamente a la permanencia del mismo, el estado civil y con el ingreso $(p<0,05)$. Conclusión: Las personas con estomas necesitan asistencia relacionada a la orientación para el autocuidado, prevención y tratamiento de sus complicaciones.

DESCRIPTORES: Estoma; Perfil de salud; Servicios de salud; Evaluación de salud; Enfermería; Estomaterapia.

\section{INTRODUÇÃO}

A pessoa com estomia é aquela que foi submetida a uma cirurgia para a confecção de uma estomia. Dentre essas pessoas, incluem-se indivíduos de todas as idades e de diferentes perfis sociodemográficos. A construção de uma estomia pode ser uma possibilidade terapêutica de sobrevivência ante a um diagnóstico clínico, na maioria das vezes, de câncer colorretal, de fundamental importância para recuperação fisiológica e reabilitação dessa pessoa ${ }^{1-3}$.

As estomias de eliminação mais frequentes são as urinários e as intestinais. As urinárias são comumente denominadas de derivações urinárias, realizadas em pessoas com doenças que envolvem a pelve renal, ureteres, bexiga e uretra, e têm por objetivo preservar a função renal. As intestinais são indicadas quando alguma parte do intestino apresenta disfunção, obstrução ou trauma. Conforme a localização anatômica, a estomia recebe denominação própria, assim, a ileostomia é no íleo e a colostomia no cólon ${ }^{4-6}$.

O planejamento do cuidado à pessoa com estomia fundamenta-se na avaliação e no monitoramento das práticas de saúde, além do conhecimento das características sociodemográficas e clínicas dos portadores de estomias, já que essa condição pode influenciar a assistência para além da demanda de cuidados específicos com a estomia, como modificar os hábitos de alimentação, o modo de vestir e a prática de atividades sexuais ${ }^{7}$.

Dada a relevância da análise de situação de saúde, dados epidemiológicos que retratam o perfil das pessoas com estomias ainda são escassos, devido, principalmente, às dificuldades de sistematização das informações de saúde 7 . A descrição das condições de saúde, doença ou adoecimento é uma medida necessária na gestão dos serviços, dos programas e das práticas do cotidiano desses serviços.

Assim, este estudo buscou analisar as condições clínicas e sociodemográficas de pessoas com estomias atendidas em seis Serviços de Atenção à Saúde da Pessoa Ostomizada (Saspo) do estado de Minas Gerais, Brasil.

\section{MÉTODOS}

Estudo transversal realizado na região oeste do estado de Minas Gerais, em 2016. Essa região abarca 54 municípios, 
com o total de 1.218.354 habitantes, e atualmente tem seis Saspo; desses, cinco são classificados como tipo I e um como tipo $\mathrm{II}^{8}$.

O Saspo do tipo I realiza ações de orientação para o autocuidado, prevenção de complicações nas estomias e fornecimento de equipamentos coletores e adjuvantes de proteção e segurança. Já o do tipo II, além de desenvolver as atividades previstas no primeiro, trata complicações que podem surgir e realiza ações de capacitação de profissionais ${ }^{6}$.

Os critérios de inclusão para a coleta de dados foram: pessoas com estomias que estavam recebendo atendimento nos Saspo I e II e que possuíam a ficha cadastral da primeira avaliação de enfermagem e médica no momento de inserção aos serviços, até o período da coleta de dados, entre janeiro e abril de 2016. Foram analisados todos os prontuários de portadores de estomias atendidas nos seis serviços, sendo os dados coletados a partir das fichas de cadastro. Essas fichas contêm informações sobre as variáveis sociodemográficas (sexo, estado civil, idade, escolaridade e renda familiar), as características das estomias (estomia, permanência, formato, diâmetro e eliminação de efluentes) e as condições dessas pessoas (realização do autocuidado, forma do abdome, tratamento oncológico e complicações das estomias).

Realizou-se análise descritiva dos dados com apresentação da distribuição de frequência para as variáveis categóricas e medidas de posição e dispersão. A variável quantitativa idade apresentou distribuição assimétrica, segundo o teste de normalidade Shapiro Wilk; assim, essa foi apresentada em mediana $\left(\mathrm{P}_{25}\right.$ e $\left.\mathrm{P}_{75}\right)$.

Para comparação de proporções, realizou-se o teste de qui-quadrado de Pearson. Ressalta-se que, para identificação das possíveis diferenças encontradas, empregou-se a análise de proporção $2 \times 2$. Nessa análise, utilizou-se a correção de Bonferroni, que altera o nível de significância (p) com intuito de evitar erros do tipo I derivados de múltiplas comparações. O nível de significância corrigido, após esse procedimento, foi de $\mathrm{p}<0,016^{9}$.

Os dados foram tabulados e analisados com auxílio dos softwares Epidata (versão 3.1. Epidata Association) e Statistical Package for the Social Sciences for Windows Student Version (SPSS), versão 19.0. O nível de significância estatística foi estabelecido em 5\%.

Este estudo foi conduzido seguindo os rigores éticos da Resolução no 466/2012, com parecer no 1.251.725 aprovado pelo Comitê de Ética em Pesquisas com Seres Humanos da Universidade Federal São João del-Rei.

\section{RESULTADOS}

Foram analisados dados de 418 pessoas com estomias cadastradas e ativas nos serviços, distribuídas em 52,1\% ( $n=218)$ no Saspo I e 47,9\% ( $\mathrm{n}=200)$ no Saspo II. A idade dessas pessoas variou de 8 meses até 97 anos, com idade mediana de 64 anos $\left(\mathrm{P}_{25}: 0 ; \mathrm{P}_{75}: 97\right)$. Mais da metade (52,9\%, $\mathrm{n}=221)$ é do sexo masculino e $51,7 \%(\mathrm{n}=216)$ são casadas.

Quanto à escolaridade, observou-se que 42,6\% ( $n=159)$ cursaram o ensino fundamental, seguidos de 28,4\% ( $\mathrm{n}=106$ ) semianalfabetos e 10,5\% ( $n=39)$ analfabetos. A renda entre um e dois salários foi a mais expressiva, com 68,8\% (n=234).

O câncer colorretal foi a principal causa de confecção de estomias, com $74,1 \%(n=310)$. Outras causas foram câncer de bexiga, de útero, doença de Crohn, gangrena de Fournier, obstrução e perfuração intestinal, representando $14,4 \%(n=60)$. Observou-se a ocorrência de 11,5\% ( $n=48)$ de pessoas com estomias sem diagnósticos definidos.

Com relação às características das estomias, os tipos mais frequentes foram colostomias esquerdas $(51,6 \%, \mathrm{n}=215)$, colostomias direitas $(18,7 \%, \mathrm{n}=78)$ e ileostomias $(17 \%$, $\mathrm{n}=71)$. Mais da metade $(56,6 \%, \mathrm{n}=237)$ possuía a estomia definitiva e $73,2 \%$ ( $n=271)$ com formato regular. Quanto ao diâmetro, medido em milímetros ( $\mathrm{mm}$ ), a maior ocorrência apresentada foi entre 21-30 mm, com 29,9\% ( $\mathrm{n}=118$ ), seguido de 28,7\% ( $\mathrm{n}=113$ ) das estomias, com 31-40 mm. Também foi verificado que mais da metade $(62,4 \%, \mathrm{n}=261)$ demonstrou ter eliminação de efluentes com a consistência pastosa.

A maioria das pessoas com estomias $(88,3 \%, \mathrm{n}=369)$ utilizava bolsas drenáveis, sendo $56,8 \%(\mathrm{n}=222)$ do tipo uma peça. Com relação ao uso de outros equipamentos e adjuvantes para o cuidado, foram registradas as indicações de pasta protetora, placa de resina sintética, cinto e, também, pó e coletor urinário, totalizando 41,4\% ( $\mathrm{n}=173)$.

Sobre as condições das pessoas com estomias, 51,9\% $(\mathrm{n}=206)$ realizavam o autocuidado com as estomias e $23,4 \%$ $(\mathrm{n}=93)$ precisavam de auxílio para realizar o cuidado. Ainda, $94,4 \%(n=368)$ não estavam acamados e 22,8\% ( $n=60)$ eram submetidos à radioterapia ou quimioterapia. Dentre as complicações mais frequentes, citam-se dermatite periestomal $(15,5 \%, \mathrm{n}=63)$, hérnia paraestomal $(11,6 \%, \mathrm{n}=48)$, prolapso $(6,8 \%, \mathrm{n}=28)$ e retração $(5,7 \%, \mathrm{n}=23)$.

A Tabela 1 apresenta a distribuição das variáveis sociodemográficas e clínicas segundo o tipo de estomia. A análise mostrou que o tipo de estomia associou-se 
significativamente com o estado civil $(\mathrm{p}=0,007)$, renda $(\mathrm{p}=0,024)$ e permanência da estomia $(\mathrm{p}=0,001)$. Após a correção de Bonferroni, observou-se maior proporção de solteiros e viúvos entre aqueles com colostomia em relação àqueles com urostomia ( $\mathrm{n}=191 ; 45,8 \%$ vs. $\mathrm{n}=98 ; 23,5 \%)$, e as pessoas com colostomia apresentaram menor renda em relação àquelas com ileostomia ( $\mathrm{n}=78 ; 18,6 \%$ vs. $\mathrm{n}=147$; $35,1 \%)$. A proporção de estomia definitiva foi maior, com $88,2 \%(n=369)$ entre aqueles com urostomia, 59,8\% $(n=250)$ com colostomia e $36,4 \%(n=152)$ com ileostomia.
A Tabela 2 aponta a distribuição percentual de variáveis sociodemográficas e clínicas segundo o tipo de Saspo e os resultados indicaram esse associou-se significativamente com a renda $(p<0,001)$, o autocuidado $(p<0,001)$ e a realização de quimioterapia ou radioterapia ( $p<0,040)$. Observou-se que as pessoas com estomia do Saspo I apresentam maior renda em relação aos da Saspo II, com a proporção de renda acima de dois salários mínimos, e que as pessoas com estomias do Saspo II apresentaram maior proporção de não realizarem o autocuidado.

Tabela 1. Distribuição percentual de variáveis sociodemográficas e clínicas segundo o tipo de estomia das pessoas com estomias da região oeste do estado Minas Gerais, $2016(n=418)$.

\begin{tabular}{|c|c|c|c|c|c|}
\hline \multirow{2}{*}{$\begin{array}{c}\text { Variáveis sociodemográficas } \\
\text { e clínicas }\end{array}$} & Total & Ileostomia & Colostomia & Urostomia & \multirow{2}{*}{ p-valor } \\
\hline & \multicolumn{4}{|c|}{ n (\%) } & \\
\hline Faixa etária (anos) & & & & & 0,362 \\
\hline Menor de 20 & $18(4,3)$ & $1(1,5)$ & $14(4,6)$ & $3(6,2)$ & \\
\hline 20 a 59 & $146(34,9)$ & $29(40,9)$ & $103(35,2)$ & $13(25,0)$ & \\
\hline 60 ou mais & $254(60,8)$ & $41(57,6)$ & $176(60,2)$ & $37(68,8)$ & \\
\hline Sexo & & & & & 0,079 \\
\hline Feminino & $194(46,5)$ & $34(47,9)$ & $143(48,8)$ & $17(32,1)$ & \\
\hline Masculino & $224(53,5)$ & $37(52,1)$ & $150(51,2)$ & $36(67,9)$ & \\
\hline Estado civil & & & & & 0,007 \\
\hline Casado ou amasiado & $246(58,9)$ & $46(64,7)$ & $159(54,2)$ & $41(76,5)$ & \\
\hline Solteiro/viúvo/outros & $172(41,1)$ & $25(35,3)^{\mathrm{ab}}$ & $134(45,8)^{a}$ & $12(23,5)^{b}$ & \\
\hline Escolaridade & & & & & 0,827 \\
\hline Sem instrução & $163(38,9)$ & $29(41,3)$ & $115(39,2)$ & $18(33,3)$ & \\
\hline Ensino fundamental & $178(42,6)$ & $32(44,4)$ & $121(41,5)$ & $25(46,7)$ & \\
\hline Ensino médio ou superior & $77(18,5)$ & $10(14,3)$ & $57(19,3)$ & $10(20,0)$ & \\
\hline Renda (salários mínimos) & & & & & 0,024 \\
\hline Até 2 & $326(77,9)$ & $46(64,9)$ & $238(81,4)$ & $40(75,0)$ & \\
\hline Mais que 2 & $92(22,1)$ & $25(35,1)^{\mathrm{a}}$ & $55(18,6)^{b}$ & $13(25,0)^{a b}$ & \\
\hline Permanência de estomia & & & & & $<0,001$ \\
\hline Definitiva & $249(59,5)$ & $26(36,4)$ & $175(59,8)$ & $47(88,2)$ & \\
\hline Temporária & $169(40,5)$ & $45(63,6)^{a}$ & $118(40,2)^{b}$ & $6(11,8)^{c}$ & \\
\hline Autocuidado & & & & & 0,452 \\
\hline Sim & $218(52,0)$ & $35(49,2)$ & $158(53,9)$ & $24(45,1)$ & \\
\hline Não & $200(48,0)$ & $36(50,8)$ & $135(46,1)$ & $29(54,9)$ & \\
\hline Pessoa com estomia acamada & & & & & 0,413 \\
\hline Não & $394(94,3)$ & $65(92,2)$ & $276(94,2)$ & $52(98,0)$ & \\
\hline $\operatorname{Sim}$ & $24(5,7)$ & $6(7,8)$ & $17(5,8)$ & $1(2,0)$ & \\
\hline Submetendo-se à quimioterapia o & oterapia & & & & 0,283 \\
\hline Não & $323(77,2)$ & $49(68,8)$ & $233(79,5)$ & $40(76,0)$ & \\
\hline Sim & $95(22,8)$ & $22(31,3)$ & $60(20,5)$ & $13(24,0)$ & \\
\hline
\end{tabular}

Valores em negrito significam $p<0,001$. ${ }^{*}$ Teste de qui-quadrado. As proporções com letras em comum na linha são iguais estatisticamente segundo a correção de Bonferroni ( $p$ > 0,05). Fonte: Serviço de Atenção à Saúde da Pessoa Ostomizada (Saspo) da região oeste do estado de Minas Gerais, 2016. 
Tabela 2. Distribuição percentual das variáveis sociodemográficas e clínicas segundo o tipo de Saspo da região oeste do estado de Minas Gerais, $2016(n=418)$.

\begin{tabular}{|c|c|c|c|c|}
\hline \multirow{2}{*}{ Variáveis sociodemográficas e clínicas } & Total & Saspo I & Saspo II & \multirow{2}{*}{ p-valor* } \\
\hline & & $\mathrm{n}(\%)$ & & \\
\hline Faixa etária (anos) & & & & 0,325 \\
\hline Menor de 20 & $18(4,3)$ & $8(3,9)$ & $10(5,1)$ & \\
\hline 20 a 59 & $146(34,9)$ & $83(38,0)$ & $62(31,1)$ & \\
\hline 60 ou mais & $254(60,8)$ & $127(58,1)$ & $128(63,8)$ & \\
\hline Sexo & & & & 0,583 \\
\hline Feminino & $194(46,5)$ & $98(45,1)$ & $96(47,8)$ & \\
\hline Masculino & $224(53,5)$ & $120(54,9)$ & $104(52,2)$ & \\
\hline Estado civil & & & & 0,663 \\
\hline Casado ou amasiado & $246(58,9)$ & $130(60,0)$ & $116(57,8)$ & \\
\hline Solteiro/viúvo/outros & $172(41,1)$ & $88(40,0)$ & $84(42,2)$ & \\
\hline Escolaridade & & & & 0,659 \\
\hline Sem instrução & $163(38,9)$ & $81(37,3)$ & $82(41,0)$ & \\
\hline Ensino fundamental & $178(42,6)$ & $93(42,8)$ & $84(42,2)$ & \\
\hline Ensino médio ou superior & $77(18,5)$ & $44(19,9)$ & $34(16,8)$ & \\
\hline Renda (salários mínimos) & & & & $<0,001$ \\
\hline Até 2 & $326(77,9)$ & $153(70,0)$ & $175(87,5)$ & \\
\hline Mais que 2 & $92(22,1)$ & $65(30,0)$ & $25(12,5)$ & \\
\hline Permanência de estomia & & & & 0,659 \\
\hline Definitiva & $249(59,5)$ & $131(60,3)$ & $116(58,1)$ & \\
\hline Temporária & $169(40,5)$ & $87(39,7)$ & $84(41,9)$ & \\
\hline Autocuidado & & & & $<0,001$ \\
\hline Sim & $218(52,0)$ & $140(64,4)$ & $78(39,2)$ & \\
\hline Não & $200(48,0)$ & $78(35,6)$ & $122(60,8)$ & \\
\hline Pessoa com estomia acamada & & & & 0,496 \\
\hline Não & $394(94,3)$ & $204(93,6)$ & $190(95,2)$ & \\
\hline Sim & $24(5,7)$ & $14(6,4)$ & $10(4,8)$ & \\
\hline Submetendo-se à quimioterapia ou radioterapia & & & & 0,040 \\
\hline Não & $323(77,2)$ & $156(71,4)$ & $164(82,1)$ & \\
\hline $\operatorname{sim}$ & $95(22,8)$ & $62(28,6)$ & $36(17,9)$ & \\
\hline
\end{tabular}

Valores em negrito significam $p<0,001$. *Teste de qui-quadrado. Fonte: Serviço de Atenção à Saúde da Pessoa Ostomizada (Saspo) da região oeste do estado de Minas Gerais, 2016.

\section{DISCUSSÃO}

A análise de dados revelou a ocorrência de pessoas com estomia com mais de 60 anos, corroborando os estudos nacionais e internacionais ${ }^{10-12}$. Os idosos têm características únicas e são mais vulneráveis às doenças crônico-degenerativas, dentre elas as neoplasias, doença de maior incidência em pessoas com idade acima de 50 anos $^{1}$. Em se tratando de idosos, os profissionais de saúde devem compreender para além da condição de estar com uma estomia, pois poderá ocorrer, conjuntamente, a presença de outras mudanças relacionadas ao envelhecimento que também podem interferir no autocuidado.

Não houve diferenças quanto ao sexo, apesar de que a maior ocorrência de estomias foi entre os homens, o que indica uma mudança no perfil de adoecimento dessas pessoas $^{10-13}$. Outros estudos, no Brasil, mostraram maior prevalência de estomias do sexo feminino até $2012^{2,5}$. Ainda em relação ao sexo, acrescenta-se que os homens parecem ter mais dificuldade com ajustamento emocional após a 
cirurgia, quando comparados com as mulheres mais idosas com estomias $^{14}$.

Neste estudo, a principal causa da confecção da estomia foi o câncer colorretal, o que condiz com a diversidade de estudos nacionais realizados com pessoas com estomias. Em termos epidemiológicos, o câncer colorretal representa a terceira neoplasia mais comum em ambos os sexos e a segunda causa de morte em países desenvolvidos ${ }^{3,10,11}$. No Brasil, o câncer colorretal é o segundo mais incidente em mulheres, com estimativa de 17.620 casos novos, e o terceiro mais incidente em homens, com 16.660 casos novos. $\mathrm{O}$ risco estimado é de 17,24 a cada 100 mil mulheres e 15,44 casos novos a cada 100 mil homens ${ }^{15}$.

No que diz respeito à escolaridade, os resultados apontaram de um lado, o predomínio de pessoas com baixa escolaridade e, de outro, que o nível de instrução não interfere no tipo de estomia realizada. Esse achado aponta para a importância das práticas de saúde voltadas para atividades promocionais que fortaleçam o autocuidado das pessoas com estomias. Sabe-se que quanto menor a escolaridade, maior o déficit de conhecimento sobre autocuidado, medidas preventivas e detecção precoce de complicações ${ }^{4}$. $\mathrm{O}$ maior nível educacional pode influenciar, ainda, nas atitudes das pessoas em relação à estomia e aos mecanismos adaptativos e, assim, facilitar sua nova condição de vida ${ }^{16}$. Nesse propósito, a educação é uma ferramenta necessária, que garante a inclusão participativa das pessoas com estomias na sociedade, no desenvolvimento de habilidades para o enfrentamento de novas adaptações perante as transformações físicas, psicológicas e sociais ${ }^{17}$.

A situação socioeconômica também pode interferir na qualidade de vida de uma pessoa com estomia ${ }^{5}$. A população com baixo poder aquisitivo poderá encontrar dificuldades na aquisição dos equipamentos e adjuvantes necessários para uma boa qualidade de vida, especialmente quando alguns desses produtos têm custo elevado e não são fornecidos pelos Saspo. Agrega-se, aos aspectos sociais, a participação da família, especialmente do cônjuge, como apoio essencial no processo terapêutico, de reabilitação e de reinserção social da pessoa com estomia ${ }^{18}$.

Um dos problemas enfrentados pelos casais diz respeito à condição de ter estomia e o quanto essa pode interferir na atividade sexual do casal. Um estudo realizado no Brasil mostrou que $36,1 \%$ mudaram suas práticas sexuais e 30,6\% cessaram as relações íntimas após a confecção da estomia ${ }^{19}$. Para os cônjuges que têm parceiro com estomia, a eliminação de gases, a presença de ruído e odor exalado pela estomia são fatores que incomodam a intimidade do casal.

Com os avanços na tecnologia, novos equipamentos coletores e adjuvantes de proteção e segurança às pessoas com estomias estão sendo desenvolvidos, com objetivo de diminuir os problemas relacionados ao vazamento de fezes, odores e gases que interferem nas atividades da vida diária e geram sentimento de medo e vergonha ${ }^{19,20}$. Ademais, torna-se essencial a familiarização com os novos dispositivos, selecionando-os adequadamente de acordo com as necessidades de cada tipo de estomia.

Quanto ao tipo de estomia, houve predominância da colostomia, seguida pela ileostomia, corroborando estudos nacionais ${ }^{10,11}$ e internacionais ${ }^{3,12}$. A colostomia resulta de uma intervenção cirúrgica realizada nos tratamentos do câncer colorretal, geralmente de caráter definitivo e, portanto, causa grande impacto na condição de vida do portador de estomia e sua família ${ }^{5,11}$.

A maior ocorrência de pessoas colostomizadas sugere o planejamento de uma assistência que consiga reduzir a presença de lesões periestomais e possibilite que essa pessoa possa realizar a irrigação, orientando quanto o tempo entre alimentação e evacuação, a condição de absorção de alimentos e medicamentos, dentre outros ${ }^{21}$.

É evidente a associação entre as estomias de caráter definitivo aos cânceres colorretal e urogenital e das temporárias ligadas aos traumas ${ }^{10,12}$. O período de permanência das estomias está diretamente relacionado com o diagnóstico de indicação de sua confecção. É temporária quando se estabelece um determinado período de tempo, o qual pode ser de meses a anos, decorrente da necessidade de proteção de uma anastomose intestinal ou de um trauma abdominal. E definitivo quando a pessoa com estomia terá que conviver com a estomia por tempo indeterminado, realizada com objetivo de substituir a perda de função esfinctérica resultante de um tratamento cirúrgico ${ }^{11,18}$.

Sobre o formato da estomia, o tipo regular reduz complicações, especialmente pela promoção da adaptação do equipamento coletor ${ }^{13}$. Nesta pesquisa, grande parte das estomias foi identificada com diâmetro de 21-30 mm. Identificar o diâmetro da estomia é essencial para o adequado plano de cuidados que possibilitará a seleção apropriada do equipamento e adjuvante e a adaptação ideal da base adesiva de barreira cutânea à estomia, evitando lesões cutâneas periestomais e promovendo maior conforto para a pessoa ${ }^{10,18}$. 
A confecção de uma estomia é potencialmente acompanhada de complicações, as quais, na maioria das vezes, são subestimadas. Estudos mostram que taxas de complicações relacionadas a estomias chegam a $60 \%{ }^{12,13}$. Das complicações pós-operatórias apresentadas na estomia e na pele, foram registradas, nos prontuários das pessoas com estomias da região estudada, dermatite, hérnia e prolapso. A origem das complicações pode ser consequência da ausência de consultas pré-operatórias, com destaque na escassez de demarcação da estomia no pré-operatório, pois a má localização dificulta o autocuidado, a visualização da pele e a troca de equipamentos ${ }^{18}$. Tais complicações podem resultar em desconforto devido ao escapamento frequente dos efluentes e à adaptação inadequada da placa na estomia.

Identificou-se, ainda, o uso de equipamento coletor drenável ou aberto do tipo sistema em uma peça pelas pessoas com estomias. No Brasil, outro estudo já demonstrou resultado semelhante em que $94,4 \%$ dessas pessoas utilizavam a bolsa de peça única, e todas as bolsas eram drenáveis ou abertas ${ }^{13}$

Cabe destacar que a indicação do sistema coletor deve ser individualizada, de maneira a considerar as características da estomia de cada pessoa, assim como seu nível de instrução. Existe maior recomendação do sistema de uma peça, por esse equipamento necessitar de menos instruções, sendo a opção mais prática para o profissional de saúde, para a pessoa com estomia e para o cuidador menos instruído. Mesmo sendo essa a melhor opção, essa população ainda considera todas as informações muito complexas e se considera incapaz de aprender, já que a maioria desse público tem baixa escolaridade $8,10,13,18$.

Constatou-se, na análise, que, além das bolsas fornecidas mensalmente pelo serviço, as pessoas com estomias recebem outros materiais adjuvantes, como placas, pós e pastas, que também são fornecidos pelo Sistema Único de Saúde (SUS), e as indicações dos equipamentos e adjuvantes dependem do tipo de estomia, da consistência do efluente e da confecção cirúrgica ${ }^{10,13}$

Os resultados apontaram que metade dos portadores de estomias realiza o autocuidado. Contudo, a abordagem utilizada nesta pesquisa não possibilitou investigar a qualidade desse cuidado. Outros estudos, realizados em diferentes regiões do Brasil, apontaram que a maioria das pessoas com estomias teve dificuldades em realizá-lo, devido à falta de orientação ou de assistência de profissionais capacitados durante essa fase do tratamento ${ }^{1,10}$. A ênfase no autocuidado tem sido descrita como alternativa para que a pessoa com estomia participe ativamente do seu próprio cuidado, estimulando a responsabilidade pela continuidade dos cuidados capazes de evitar as complicações e alcançar a reabilitação.

Embora houvesse significância estatística entre as associações das características sociodemográficas e clínicas com o tipo de estomia, assim como dos tipos de Saspo com o autocuidado e a realização do tratamento oncológico, esses resultados não apresentam significância clínica entre si, ou seja, a interpretação dos resultados dos estudos mostrou resultados estatisticamente significativos, mas não clinicamente significativos, por não terem utilidade na prática clínica diária.

Foi considerado, como limitação deste estudo, o fato de que, por serem pesquisadas apenas pessoas com estomias vinculadas aos serviços públicos de saúde (SUS), não se pode inferir o real perfil dessa população na região. Outras limitações foram relacionadas à incompletude de campos em branco encontrados nas fichas da primeira avaliação da equipe dos Saspo, no momento de inserção das pessoas com estomias aos serviços, e no formulário padronizado pela Secretária de Estado de Saúde de Minas Gerais (SES/ MG), que precisa ser atualizado.

Essas informações podem subsidiar a elaboração de propostas de estratégias de ações de saúde com vistas a melhorar as práticas de saúde e, consequentemente, a qualidade do cuidado às pessoas com estomias.

\section{CONCLUSÃO}

As pessoas com estomias atendidas nos Saspo avaliados são, na maioria, idosas, com baixo nível de escolaridade e baixa renda salarial. A neoplasia maligna colorretal foi a principal causa e a estomia do tipo colostomia definitiva a de maior ocorrência. Os equipamentos coletores mais utilizados são os do tipo drenáveis, sistema uma peça, sendo utilizados adjuvantes do tipo placas, pastas e pós.

Este estudo apresentou, como pontos positivos: revelar os resultados sobre as características clínicas e sociodemográficos das pessoas com estomias, como informação necessária para a tomada de decisão ante a organização dos serviços locais; apontar para o aprimoramento da qualidade das informações dos prontuários; orientar sobre a necessidade de rever o formulário padrão utilizado pela SES/MG, bem como a 


\section{CONTRIBUIÇÃO DOS AUTORES}

formação dos profissionais envolvidos nas atividades dos serviços, melhorando os registros da assistência prestada a essa clientela. As pessoas com estomias necessitam de assistência relacionada à orientação para o autocuidado, prevenção e tratamento de complicações das estomias.
Conceitualização,Moura RRA; Guimarães EAA e Moraes JT; Metodologia, Guimarães EAA e Moraes JT; Investigação, Moura RRA e Moraes JT; Redação - Primeira versão, Moura RRA; Guimarães EAA e Moraes JT; Redação - Revisão \& Edição, Guimarães EAA e Moraes JT.

\section{REFERÊNCIAS}

1. Silva J, Sonobe HM, Buetto LS, Santos MG, Lima MS, Sasaki VDM. Estratégias de ensino para o autocuidado de estomizados intestinais. Rev Rene (Online). 2014;15(1):16673. https://doi.org/10.15253/2175-6783.2014000100021

2. Melotti LF, Bueno IM, Silveira, GV, Silva MEN, Fedosse E. Characterization of patients with ostomy treated at a public municipal and regional reference center. J Coloproctol (Rio J). 2013;33(2):70-4. https://doi.org/10.1590/s2237-936320130 $\underline{00200005}$

3. Sasaki VDM, Pereira APS, Ferreira AM, Pinto MH, Gomes J. Health care service for ostomy patients: profile of the clientele. J Coloproctol (Rio J). 2012;32(3). https://doi. org/10.1590/s2237-93632012000300005

4. Lenza NFB, Sonobe HM, Buetto LS, Santos MG, Lima MS. O ensino do autocuidado aos pacientes estomizados e seus familiares: uma revisão integrativa. Rev Bras Promoç Saúde, Fortaleza. 2013;26(1):139-45.

5. Luz ALA, Luz MHBA, Antunes A, Oliveira GS, Andrade EMLR, Miranda SM. Perfil de pacientes estomizados: revisão integrativa da literatura. Cultura de los Cuidados. 2014;18(39):115-23. https://doi.org/10.7184/cuid.2014.39.13

6. Ministério da Saúde (BR). Secretaria de Atenção à Saúde. Portaria $n^{\circ}$ 400, de 16 de novembro de 2009. [Internet]. Brasília, DF; 2009. Disponível em: http://www.ans.gov.br/ images/stories/noticias/pdf/p sas 4002009 ostomizados.pdf

7. Moraes JT, Amaral CFS, Borges EL, Ribeiro MS, Guimarães EAA. Serviços de atenção ao estomizado: análise diagnóstica no estado de Minas Gerais, Brasil. Cad Saúde Colet, Rio de Janeiro. 2014;22(1):101-8. https://doi.org/10.1590/1414$462 \times 201400010015$

8. Ribeiro MS, Borges EL. Linha de cuidados da pessoa estomizada. Belo Horizonte: Secretaria de Estado de Saúde de Minas Gerais; 2015.

9. Field A. Descobrindo a estatística usando o SPSS. 2a ed. Porto Alegre: Artmed; 2005.

10. Salomé GM, Carvalho MRF, Junior MRM, Mendes B. Profile of ostomy patients residing in Pouso Alegre city. J Coloproctol (Rio J). 2015;5(2):106-12. https://doi.org/10.1016/j.jcol.2015. $\underline{02.002}$

11. Neto MAFL, Fernandes DOA, Didoné EL. Epidemiological characterization of ostomized patients attended in referral center from the city of Maceió, Alagoas, Brazil.
J Coloproctol (Rio J). 2016;36(2):64-8. https://doi.org/10.1016/j. jcol.2014.08.016

12. Joyce P. Characteristics of the patient with an ostomy. J Wound Ostomy Continence Nurs. 2011;38(3):271-9. https:// doi.org/10.1097/WON.0b013e3182152bbf

13. Miranda SM, Luz MHBA, Sonobe HM, Andrade EMLR, Moura ECC. Caracterização sociodemográfica e clínica de pessoas com estomia em Teresina. ESTIMA, Braz J Enterostomal Ther. 2016;14(1):29-35. https://doi.org/10.5327/Z1806-31442016 $\underline{00010005}$

14. Skeps R, McMullen CK, Wendel CS, Bulkley J, Grant M, Mohler J et al. Changes in body mass index and stoma related problems in the elderly. J Geriatr Oncol. 2013;4(1):84-9. https://doi.org/10.1016/i.jgo.2012.10.172

15. Ministério da Saúde (BR). Instituto Nacional de Câncer. Estimativa 2016: incidência de câncer no Brasil [Internet]. Brasília, DF: 2016. Disponível em: https://www.inca.gov. br/campanhas/dia-nacional-de-combate-ao-cancer/2015/ estimativa-2016-incidencia-de-cancer-no-brasil

16. FanCheng MD, Ai-fengMeng BD, Li-Fang Yang BD, Yi-nan Zhang, BD. The correlation between ostomy knowledge and self-care ability with psychosocial adjustment in Chinese patients with a permanent colostomy: a descriptive study. Ostomy Wound Manage. 2013;59(7):35-8.

17. Grant Ma, Corkle RM, Hornbrook MC, Wendel SC, Krouse R. Development of a chronic care ostomy self-management program. J Cancer Educ. 2013;28(1):70-8. https://doi. org/10.1007/s13187-012-0433-1.

18. Silva AC, Silva GNS, Cunha, RR. Caracterização de pessoas estomizadas atendidas em consulta de enfermagem do Serviço de Estomaterapia do Município de Belém-PA. ESTIMA, Braz J Enterostomal Ther. 2012;10(1).

19. Silva AL, Kamada I, Sousa JB, Vianna AL, Oliveira PG. Singularidades da convivência do cônjuge e seu parceiro estomizado. ESTIMA, BrazJ Enterostomal Ther. 2016;14(2):6875. https://doi.org/ 10.5327/Z18063144201600020004

20. Diebold L. Stoma and shame: engaging affect in the adaptation to a medical device. Aust J Adv Nurs. 2016;34(1):32-41.

21. Moraes JT, Assunção RS, Sá FS, Lessa ER, Corrêa LS. Perfil de pessoas estomizadas de uma região de saúde mineira. Enferm Foco. 2016;7(2):22-6. 